Chapman University

Chapman University Digital Commons

Athletic Training Faculty Articles and Research

Athletic Training

$3-2014$

\title{
Thermoregulatory, Cardiovascular, and Perceptual Responses to Intermittent Cooling During Exercise in a Hot, Humid Outdoor Environment
}

Michelle A. Cleary

Chapman University, cleary@chapman.edu

Michelle G. Toy

Dellarmine College Preparatory

Rebecca M. Lopez

University of South Florida

Follow this and additional works at: http://digitalcommons.chapman.edu/athletic_training_articles

Part of the Exercise Science Commons

\section{Recommended Citation}

Cleary, M.A., Toy, M.G., Lopez, R.M., 2014. Thermoregulatory, Cardiovascular, and Perceptual Responses to Intermittent Cooling During Exercise in a Hot, Humid Outdoor Environment: Journal of Strength and Conditioning Research 28, 792-806. doi:10.1519/ JSC.0b013e3182a20f57 


\section{Thermoregulatory, Cardiovascular, and Perceptual Responses to Intermittent Cooling During Exercise in a Hot, Humid Outdoor Environment}

\section{Comments}

This is a non-final version of an article published in final form in

Cleary, M.A., Toy, M.G., Lopez, R.M., 2014. Thermoregulatory, Cardiovascular, and Perceptual Responses to Intermittent Cooling During Exercise in a Hot, Humid Outdoor Environment: Journal of Strength and Conditioning Research 28, 792-806. DOI: 10.1519/JSC.0b013e3182a20f57

\section{Copyright}

Lippincott, Williams \& Wilkins 


\title{
TITLE PAGE
}

Running Head: Intermittent cooling during exercise in the heat

Thermoregulatory, Cardiovascular, and Perceptual Responses to Intermittent Cooling during Exercise in a Hot, Humid Outdoor Environment

\author{
Michelle A. Cleary PhD, ATC, CSCS ${ }^{1}$ \\ Michelle G. Toy MS, ATC, CSCS ${ }^{2}$ \\ Rebecca M. Lopez PhD, ATC, CSCS ${ }^{3}$
}

1. Athletic Training Education Program, College of Educational Studies, Chapman University, Orange, CA

2. Head Strength and Conditioning Specialist, Dellarmine College Preparatory, San Jose, CA;

3. Department of Orthopedics \& Sports Medicine, University of South Florida, Tampa, FL

Direct correspondence to:

Michelle A. Cleary, PhD, ATC, CSCS Associate Professor

Athletic Training Education Program

College of Educational Studies

Chapman University

Phone: (714)628-2797

Email: cleary@chapman.edu 
Intermittent cooling during exercise in the heat

Page 1

\title{
Thermoregulatory, Cardiovascular, and Perceptual Responses to Intermittent Cooling during Exercise in a Hot, Humid Outdoor Environment
}

\begin{abstract}
Decreasing core body temperature during exercise may improve exercise tolerance, facilitate acclimatization, and prevent heat illness during summer training. We sought to evaluate the effectiveness of intermittent superficial cooling on thermoregulatory, cardiovascular, and perceptual responses during exercise in a hot-humid environment. We used a randomized, counterbalanced, repeated measures investigation with two conditions (Control, Cooling) during exercise and recovery outdoors on artificial turf in a hot, humid tropical climate in the sun $\left(\mathrm{WBGT}_{0}=27.0 \pm 0.8^{\circ} \mathrm{C}\right.$, range $\left.=25.8-28.1^{\circ} \mathrm{C}\right)$ and in the shade $\left(\mathrm{WBGT}_{0}=25.4 \pm 0.9^{\circ} \mathrm{C}\right.$, range $=24.3-$ $26.8^{\circ} \mathrm{C}$ ). Participants were 10 healthy males (age $=22.6 \pm 1.6 \mathrm{y}$; height $=176.0 \pm 6.9 \mathrm{~cm}$; mass $=76.5 \pm 7.8 \mathrm{~kg}$; body fat $=15.6 \pm 5.4 \%$ ) who wore shorts and t-shirt (Control) or "phase change cooling" vest (Cooling) during 5-min rest breaks during 60 min of intense American football training and conditioning exercises in the heat and 30 min of recovery in the shade. Throughout, we measured core $\left(T_{\text {gi }}\right)$ and skin ( $\left.T_{\text {chest }}\right)$ temperature, heart rate $(H R)$, thermal and thirst sensations, and rating of perceived exertion (RPE). We found significant $(p \leq 0.001)$ hypohydration (-2.1\%) occurred; for $\mathrm{T}_{\text {gi }}$ we found no significant differences between conditions $(p=.674)$ during exercise; and progressive decreases during recovery $(p<.001)$. For $\Delta \mathrm{T}_{\text {gi }}$ we found no significant $(p=.090)$ differences. For $T_{\text {chest }}$ we found significantly $(p<0.001)$ decreased skin temperature for the Cooling condition $\left(T_{\text {chest }}=31.85 \pm 0.43^{\circ} \mathrm{C}\right)$ compared to the Control condition $\left(T_{\text {chest }}=34.38 \pm 0.43^{\circ} \mathrm{C}\right)$ during exercise and significantly $(p<0.001)$ lower skin temperature in the Cooling condition $\left(\mathrm{T}_{\text {chest }}=31.24 \pm 0.47^{\circ} \mathrm{C}\right)$ compared to the Control condition $\left(\mathrm{T}_{\text {chest }}=33.48 \pm 0.47^{\circ} \mathrm{C}\right)$ during recovery. For HR we found no significant difference $(p=0.586)$ between the conditions during exercise; however we did find however we did find significantly $(p<0.001)$ lower HR during recovery. Thermal sensations were significantly $(p=0.026)$ decreased in the Cooling ( $4.4 \pm 0.2$ points) compared to the Control (5.0 \pm 0.2 points) condition, but not for other perceptual responses. The cooling effects of "phase change cooling" material were effective in reducing skin temperature but did not sufficiently reduce core body temperature or cardiovascular strain.
\end{abstract}

Key Words: Exertional heat illness, intermittent cooling, heat strain 


\section{Thermoregulatory, Cardiovascular, and Perceptual Responses to Intermittent Cooling during Exercise in a Hot, Humid Outdoor Environment}

\section{INTRODUCTION}

Athletes training and competing in hot, humid environments are particularly susceptible

to exertional heat illnesses $(\mathrm{EHI})$ such as heat cramps, heat exhaustion, and heat stroke.

Training and competing in hot environments pose real challenges to athletes as difficulty arises

in avoiding hyperthermia. The challenge to exercise in the heat is maintaining body water

stores within safe limits while losing sweat for evaporative cooling and continuing performance

at a level to maintain competitiveness without compromising personal safety. Exercise

sustained at a sufficient intensity and duration may elevate core body temperature to the upper

limit of its thermoregulatory zone. Thermoregulatory controls override those of body water regulation so the main concerns are the system errors when core temperature rises beyond its safe limit and the physiological factors that protect the athlete from heat injury begin to fail under uncompensable heat gain.(30) In football, August is the month with the highest incidence of EHIs with $88 \%$ of $\mathrm{EHI}$ (rate $=8.95 / 1000$ athlete exposures) reported during this time.(11) This high incidence is due, in part, to the nature of pre-season practices beginning in July or August, which are the hottest times of the year in most regions of the United States. The National Collegiate Athletic Association (NCAA)(12) implemented legislation allowing a gradual progression in the use of protective equipment over the pre-season acclimatization period. Other governing organizations [American College of Sports Medicine,(3) National Athletic Trainers' Association(6)] have issued position statements that provide guidelines for acclimatizing athletes to exercise in hot, humid environments including limiting exposure to the environment, reducing protective equipment covering the skin, increasing fluid consumption, and reducing work to rest ratios. Controversy exists relating to the "critical internal" temperature limitations to exercise in the heat. Methods of preventing EHI may include blunting the exercise-induced increased body temperature (hyperthermia) to limit the critical internal 
64 temperature to less than $40{ }^{\circ} \mathrm{C}$ when the central fatigue mechanism is initiated.(20) However,

65 "self-pacing", runners have been demonstrated to be able to sustain running velocity despite

66 rectal temperature $>40{ }^{\circ} \mathrm{C}$ providing evidence against $40{ }^{\circ} \mathrm{C}$ representing a "critical" core

67 temperature limit to performance. Although critical internal temperature limits may vary from

68 individual to individual, other factors such as hydration status, previous exercise exposure to hot

69 environments, over-zealousness/refusal to pace, or underlying illness may contribute to the

70 development of EHI when athletes reach hyperthermic temperatures.

$71 \quad$ Athletes and coaches are often willing to try new products to help improve or maintain

72 athletic performance when training or competing in hot environments. A greater understanding

73 of the delicate balance between effective warm-up and the thermoregulatory demand to

74 exercising in the heat is needed. New cooling garments have been marketed to the general

75 population to address cooling the body in hot conditions and these garments are now being

76 marketed to the athletic population. These cooling garments are constructed of "phase change"

77 materials that absorb or release latent heat have recently been marketed as thermal-regulated

78 clothing.(17) Cooling vests with phase change materials on a heated manikin in a climactic

79 chamber demonstrated that the cooling rates are dependent on temperature gradient, mass,

80 and covered area.(18) Intermittent, microclimate cooling provided favorable heat dissipation by

81 conduction and reduced heat strain comparable to continuous cooling during exercise-heat

82 stress in chemical protective clothing.(8) Participants wearing three different cooling vests

83 during rest, stretch, warm-up and recovery had reduced sweat rate, core and skin temperatures

84 during exercise, and perceptions of the thermal state and skin wetness demonstrated changes

85 to greater levels of satisfaction.(32) On the other hand, the combination of wearing a cooling

86 jacket and water intake was demonstrated to enhance exercise endurance performance in a

87 warm environment; however, core temperature at exhaustion was not different.(21) A previous

88 investigation by the current authors also did not find significant differences between a cooling

89 vest and control in hyperthermic individuals during recovery from exercise in the heat.(25) 
90 Similarly, other researchers found that 10-min, halftime cooling application failed to elicit

91 differences in sweat loss, core and mean skin temperature and rating of thermal sensation; (15,

92 23) although psychological assessment revealed a dramatic placebo effect from the cooling

93 application confounding these researcher's results.(23) Superficial cooling may help athletes

94 feel better during exercise in the heat and may prevent dangerous hyperthermia from

95 developing.

96 To minimize the risk of heat-related illness during exercise in the heat, an applicable

97 method is needed to reduce elevations on core body temperature.(34) Prevention of heat-

98 related illness may be accomplished by the use of cooling vests intermittently during exercise

99 which may attenuate cardiovascular strain and fatigue. During exercise in hot environments in

100 sports where periodic rest breaks are allowed, such as American football, soccer, hockey or

101 recreational jogging a practical method to reduce dangerous elevations in core body

102 temperature are needed. Coaches and athletes need a practical, cooling strategy applicable to

103 several sporting regulations, which is low cost, easy to use, light-weight and which enhances

104 cooling of the body core during and following exercise.(32) Athletes may benefit from a new

105 superficial cooling vest that is composed of phase change material. These cooling vests re

106 designed to be worn during outdoor training reportedly deliver a constant temperature for up to

1073 hours with no impact from ambient temperature or humidity (according to the manufacturer's

108 website and promotional materials). These phase change cooling materials are re-usable and

109 recharged by submersing the vest in ice water or placing it in a refrigerator or freezer. Few

110 studies have been conducted to examine thermoregulation with intermittent cooling with phase

111 change materials during short-duration, high-intensity intermittent exercise.(27) Therefore, the

112 purpose of the study was to determine the thermoregulatory, cardiovascular, and perceptual

113 response to intermittent, superficial cooling with phase change materials during exercise and

114 recovery in a hot, humid environment.

115 
116

117

118

119

120

\section{METHODS}

\section{Experimental Approach to the Problem}

A randomized, counterbalanced, repeated measures investigation with two experimental

121 conditions (cooling, control,) was utilized for this investigation (Figure 1). Participants

122 completed the two randomly assigned conditions in a randomized, counterbalanced order

123 separated by at least $24 \mathrm{hr}$. Since August is the month with the most EHI occurrences(11), the investigation occurred outdoors in July-August. We collected data on a tropical island on synthetic turf in a hot, humid climate (Figure 2 ) in the sun $\left(\mathrm{WBGT}_{0}=27.0 \pm 0.8^{\circ} \mathrm{C}\right.$, range $=25.8$ $\left.-28.1^{\circ} \mathrm{C}\right)$ and in the shade $\left(\mathrm{WBGT}_{0}=25.4 \pm 0.9^{\circ} \mathrm{C}\right.$, range $\left.=24.3-26.8^{\circ} \mathrm{C}\right)$. These

127 environmental conditions are considered "Red Flag" conditions according to the NATA Position 128 Statement on Exertional Heat IIIness (6) and the ACSM Position Stand on Exertional Heat 129 Illness during Training and Competition (3). In these conditions everyone should be aware of 130 injury potential, individuals at risk should not compete, rest-to-work ratio should include 5- to 10131 min rest and fluid breaks every 20 to $30 \mathrm{~min}$; and practice should be in shorts with helmets and 132 shoulder pads (not full equipment).(6) Data collection occurred on 11 days over a three-week 133 period in July-August 2010 with exercise testing scheduled at a consistent time between 2:00 134 and 6:00 pm with at least $24 \mathrm{hr}$ between testing sessions.

135 Our conditions were sufficiently stressful to simulate typical American football pre136 season training in hot, humid conditions (Figure 2). Subjects were required to exercise 137 intensely (heart rate of $85-95 \%$ of age-predicted maximum) and to reach a criterion minimum 138 core temperature $\left(38.0^{\circ} \mathrm{C}\right)$. Each data collection session consisted of a warm-up, followed by $13960 \mathrm{~min}$ of intense exercise in the heat with 5-min rest breaks every $20 \mathrm{~min}$, followed by $30 \mathrm{~min}$ 140 of recovery in the shade. During the 5-min rest breaks and continuously during $30 \mathrm{~min}$ of 141 recovery, participants wore the randomly assigned experimental superficial cooling garment or a 142 t-shirt (see Instruments for more details). We collected data during six time-matched periods 143 (Figure 1) corresponding to the beginning and end of each 5 min break provided and every 5 
144 min during 30 min of recovery. Meals, snacks, and hydration status were controlled for each trial by asking participants to maintain a consistent diet prior to each data collection session.

\section{Main Outcome Measures}

Our main outcome measures were quantified during rest breaks and period. We assessed thermoregulatory and cardiovascular responses: core body temperature $\left(T_{\text {gi }}\right)$, chest skin temperature $\left(T_{\text {chest }}\right)$, and heart rate $(H R)$ as main outcome measures. For 3-days prior to the study, we measured participants' hydration status to determine the baseline euhydrated condition. In addition, before and after each data collection session, we determined hydration 154 status consisting of: sweat rate, volume of fluid consumed $\left(F_{\text {vol }}\right)$, body mass loss $(B M L)$, urine 155 volume $\left(\mathrm{U}_{\mathrm{vol}}\right)$, urine color $\left(\mathrm{U}_{\mathrm{col}}\right),(2)$ and urine specific gravity $\left(\mathrm{U}_{\mathrm{sg}}\right)$. We also assessed perceptual sensations consisting of: the thermal sensations scale,(34) thirst sensations scale,(16) and the Borg rating of perceived exertion (RPE) scale.(7) An environmental symptoms questionnaire $(E S Q)(31)$ was used to identify symptoms of heat illness. Environmental conditions were recorded 15-min intervals throughout data collection with wet bulb globe temperature outdoors (WBGT $T_{0}$ in the direct sun and in the shade.

\section{Subjects}

We purposefully sampled 16 male participants from a pool of college students meeting the inclusionary criteria and 10 were included in data analysis $($ age $=22.6 \pm 1.6 \mathrm{yr}$; height $=$ $176.0 \pm 6.9 \mathrm{~cm}$; mass $=76.5 \pm 7.8 \mathrm{~kg}$; body fat $=15.6 \pm 5.4 \%)$. Six participants were excluded from data analysis because either they did put forth enough effort to reach the $\mathrm{T}_{\mathrm{gi}}>38.0^{\circ} \mathrm{C}$ threshold or the temperature capsule did not reach the duodenum in time for data collection. We recruited participants who were acclimatized to the environmental conditions by exercising outdoors in the tropical climate in which data collection occurred. Participants were screened by the investigators and included in the investigation if the following criteria were met: (1) had no chronic health problems; (2) exercised outdoors a minimum of 30 min per a day, 3 days a week; 
173 (3) had no previous history of heat stroke in the past 3 years; (4) had no previous history of 174 cardiovascular, metabolic, or respiratory disease; (5) were not febrile; and/or (6) were not 175 currently taking any supplements or drugs that influence thermoregulation. All subjects read 176 and signed the informed consent form before participation, and the University's Institutional 177 Review Board approved this investigation.

178

179

\section{Instruments}

Cooling Vest. The cooling vest (Cool58 ${ }^{\mathrm{TM}}$ Phase Change Vest, Polar Products, Inc., Akron, $\mathrm{OH}$ ) is marketed by the manufacturer as an "evaporative, phase-change" cooling vest that stores water in special polymers built throughout the vest with a thin layer of fabric between 184 the water storage compartments and the body (Figure 3). These vests are charged by soaking them in an ice water for at least 10 min prior to use. Following manufacturers' instructions, the vest was submerged and soaked in ice water maintained at $2.8 \pm 1.0^{\circ} \mathrm{C}$ (measured using submerged thermometer), which was maintained by adding more ice added as necessary. When removed the vest from the ice slush, allowed it to drip for 1-2 min then placed it directly on the skin. The vest was fitted to the participant and placed directly on the skin with selfadhesive straps to hold the material close to the skin (Figure 3).

Perceptual Scales. Perceptual sensations were measured using three scales: thermal, thirst, RPE, and an ESQ. The thermal sensations scale (33) was used to assess participants' perception of heat or cold and included a 9-point rating scale with $0=$ Unbearably Cold and $8=$ Unbearably Hot. The thirst sensations scale (16) was used to determine participants' level of thirst and consisted of a 9-point rating scale with $1=$ Not Thirsty At All and $9=$ Very, Very Thirsty. The RPE scale (7) was used to assess participants' perceived level of physical effort or exercise intensity and consisted of a 15-point scale (6 to 20) with $7=$ Very, Very Light and $19=$ Very, Very Hard. Finally, the ESQ (31) was used to identify symptoms of heat illness. The ESQ consisted of 14 questions including: "I felt lightheaded, I had a headache, I felt dizzy", etc. 
200

201

202

203

204

\section{Data Collection Procedures}

Familiarization Session

Following screening and enrollment, participants were familiarized with the data collection procedures by reporting to the Human Performance Laboratory where investigators recorded participant physical characteristics: body mass, height, age, BMI, body surface area (BSA), and body composition (skinfolds). Each participants' baseline euhydrated condition was determined 3 days prior to data collection and verified before starting the experiment. Heart rate training range was predicted using the $\mathrm{HR}$ maximum score and multiplying it by the percent of HR maximum (85-95\%). Before returning to the laboratory the following day for data collection, participants were provided with instructions for preparing for the experimental protocol: (1) perform only activities of daily living during the $24 \mathrm{hr}$ before each experiment; (2) consume a consistent diet, including hydration behaviors, before each data collection session to reduce the effects of different nutrient intake on exercise performance; and (3) bring a dry change of clothes to each data collection session.

\section{Experimental Protocol}

Participants arrived at the laboratory $2 \mathrm{hr}$ before beginning the exercise to swallow the ingestible temperature capsule (VitalSense ${ }^{\circledR}$, Philips Respironics, Inc., Bend, OR) and allow for it to pass into the duodenum. Participants also provided a urine sample at this time for hydration status testing using a digital clinical refractometer (Pen-Urine SG, Atago USA, Inc., Bellevue, WA). After voiding, participants were weighed on a digital platform scale (Model PS6600 ST, Befour Inc., Saukville WI) wearing only shorts then fitted with a HR monitor (Model FT1, Polar-Electro Inc. Lake Success, NY). Due to the limitation of the expense of the dermal patches, chest skin temperature was selected as a representative sample of skin exposed to the cooling vest. The skin on the left chest at the mid-clavicular line and mid-way between the clavicle and the nipple was rubbed with an alcohol pad (shaved, if necessary) and the dermal 
patch (VitalSense ${ }^{\circledR}$, Philips Respironics, Inc., Bend OR) was then attached to the chest.

Participants wore their own t-shirt, shorts, socks, and running shoes and were asked to wear the same or similar clothes for each data collection session.

Warm-Up and Anaerobic Exercise. Once prepared, participants then walked to the outdoor research area in the shade adjacent to the synthetic turf practice field. After resting seated for at least $5 \mathrm{~min}$, investigators recorded baseline measures of $T_{\text {gi }}, T_{\text {chest }}, H R$, thirst sensations, thermal sensations, RPE, and ESQ. At 15-min intervals throughout data collection, investigators monitored environmental conditions using the thermal environment monitor (Model 36, QUESTemp Inc., Oconomowoc, $\mathrm{WI}$ ) attached to a tripod set at 36 in above the synthetic turf and allowed to equilibrate for at least 5 min. After recording the data, the monitor was moved and equilibrated in the shade. In addition, turf surface temperature/humidity in the sun and ground temperature/humidity in the shade were measured by placing the wand of a temperature/humidity monitor (Model 11-661-21, Thermo-Fisher Scientific, Inc., Pittsburgh, PA) on the ground for at least $10 \mathrm{~min}$.

Participants were lead through a standardized exercise protocol for 60 min with 244 designed to achieve the following goals: 1) maintain an average heart rate of $85-95 \%$ of age245 predicted maximum, using the equation $\left(191.5-0.007 \times a^{2} e^{2}\right)(10,19)$, and 2$)$ reach a threshold $246 \mathrm{~T}_{\mathrm{gi}}$ criteria of $\geq 38.0^{\circ} \mathrm{C}$ during the exercise session. Participants who reached or exceeded $40^{\circ} \mathrm{C}$ 247 were allowed to continue if desired and monitored for signs or symptoms of EHI. One 248 participant exceeded the $40{ }^{\circ} \mathrm{C}$ safety limit and was required to rest until his $T_{\text {gi }}$ decreased to a 249 safer level. No participant exhibited signs or symptoms of EHI during the experiment. The 250 exercise protocol consisted of a supervised training program with a 12 min warm-up of dynamic 251 stretching exercises immediately followed by $60 \mathrm{~min}$ of a variety of standard football speed and 252 agility drills (Table 1). The exercises were designed to mimic the first half of an NFL-style 253 football practice with non-contact training and conditioning activities. To simulate the intensity 254 and duration of an actual football practice, exercise intensity was monitored by the participants 
4255

and recorded by the investigators at the end of each 20 min interval using the HR monitors.

Participants were asked to put forth a maximum effort for the duration of each interval and to maintain an average of $85-95 \%$ of HR maximum during the drills.

Experimental Conditions. Participants assigned to the Control condition remained seated with their t-shirts on in the shade throughout the 5-min rest breaks and recovery.

Participants assigned to the Cooling condition were asked to remove their t-shirt, put on the charged cooling vest (maintained at a temperature $=2.8 \pm 1.0^{\circ} \mathrm{C}$ ), and remain seated throughout the 5-min rest break. To avoid the confounding effects of dehydration in both conditions, participants were permitted to drink cold water ad libitum from individual water bottles at the end of each rest break after data were collected (to avoid artificially affecting the ingested thermistor in the gastrointestinal tract). The amount of fluid consumed was recorded for data analysis.

Recovery. Upon completion of the exercise protocol, participants moved to the adjacent research area in the shade for 30 min of seated recovery. In the Control condition participants rested in a seated position wearing their t-shirt. In the Cooling condition participants rested wearing the charged cooling vest. The vest was not removed until $\mathrm{T}_{\mathrm{gi}}$ reached baseline levels. At 5-min intervals during recovery, we assessed $T_{\text {gi }}$, $T_{\text {chest }}, \mathrm{HR}$, and perceptual sensations. Following recovery, participants toweled off, changed into dry clothes, and were weighed. We asked participants to refrain from voiding until weighed then we collected $\cup_{\mathrm{vol}}$ and performed urinalysis consisting of $U_{c o l}$ and $U_{s g}$.(2) Participants were provided with snacks (pretzels, bananas) and were required to rehydrate with at least $500 \mathrm{~mL}$ of cold water before leaving the laboratory.

\section{Statistical Analyses}

The treatment effects were evaluated using a randomized, crossover design. All data were statistically analyzed using separate 2-way repeated-measures (condition $x$ time) analysis of variance (ANOVA) for exercise and recovery and were reported as mean \pm SD. The 
282 difference between pre- and post-tests ( $\Delta=$ pre-post) were analyzed with a 2X8 ANOVA with repeated measures on the second factor. When statistical differences were found, the Tukey LSD test for post hoc analysis was used and post hoc t-tests with Bonferroni corrections were used to determine pairwise differences among time periods. Estimates of effect size were reported as partial $\eta^{2}$ value and power was reported as $1-\beta$ for each parameter. Pearson correlations were used to analyze the relationship between the thermoregulatory/cardiovascular responses and the perceptual responses for the end of exercise. Descriptive statistics were calculated for participant's physical characteristics, environmental conditions, and hydration status. Occasional missing data points were replaced with the series mean. For all analyses the $\alpha$ level was set at 0.05 and statistical software (SPSS Statistics 20; IBM, Inc., Chicago, IL) was used.

\section{RESULTS}

\section{Thermoregulatory and Cardiovascular Responses}

The $T_{g i}$ data from the intermittent cooling during exercise breaks resulted in a blunted or flattened thermoregulatory response for the Cooling condition (Figure 4). For $T_{g i}$ during exercise we found no significant differences between conditions $\left(F_{1,18}=0.183, p=.674\right.$, partial $\eta^{2}=.010,1$ $\beta=.069)$; however we did find a significant interaction effect $\left(F_{5,90}=2.77, p=.022\right)$ with post hoc testing revealing a significant ( $p=.010,95 \% \mathrm{Cl}=.057-.595)$ decrease in $\mathrm{T}_{\text {gi }}$ during Break 3 (pretest $=38.50 \pm .13^{\circ} \mathrm{C}$ vs. post-test $=38.18 \pm .13^{\circ} \mathrm{C}$ ) for both conditions. For $\mathrm{T}_{\text {gi }}$ during recovery, we found no significant differences between conditions $\left(F_{1,18}=0.590, p=.452\right.$, partial $\eta^{2}=.032,1$ $\beta=.113)$ but we did find a significant main effect for test $\left(F_{5,90}=6.681, p<.001\right)$ with $T_{g i}$ progressively decreasing during recovery for both conditions. For $\Delta \mathrm{T}_{\mathrm{gi}}$ we found no significant interaction or main effect for condition $\left(F_{1,18}=2.243, p=.152\right.$, partial $\left.\eta^{2}=.111,1-\beta=.294\right)$. Cooling rate revealed no significant interaction $\left(\mathrm{F}_{8,144}=1.760, p=.090\right.$, partial $\left.\eta^{2}=.089,1-\beta=.741\right)$ or main effects. 
310 The $T_{\text {chest }}$ data from the intermittent cooling during exercise resulted in dramatic

decreases in the Cooling condition (Figure 5). For $\mathrm{T}_{\text {chest }}$ during rest breaks, we found a significant interaction effect $\left(F_{5,90}=25.853, p<.001\right)$ with significantly $(p<0.001)$ decreased skin temperature for the Cooling condition $\left(T_{\text {chest }}=31.85 \pm 0.43^{\circ} \mathrm{C}\right)$ compared to the Control condition $\left(\mathrm{T}_{\text {chest }}=34.38 \pm 0.43^{\circ} \mathrm{C}\right)$. For $\mathrm{T}_{\text {chest }}$ during recovery, we found a significant interaction effect $\left(F_{5,90}=12.671, p<.001\right)$ with significantly $(p<0.001)$ lower skin temperature in the Cooling condition $\left(T_{\text {chest }}=31.24 \pm 0.47^{\circ} \mathrm{C}\right)$ compared to the Control condition $\left(T_{\text {chest }}=33.48 \pm 0.47^{\circ} \mathrm{C}\right)$. For the difference in $\Delta T_{\text {chest }}$ we found a significant interaction effect $\left(F_{5,90}=17.189, p<.001\right)$ with $\Delta T_{\text {chest }}$ significantly $\left(F_{1,18}=43.038, p<.001\right)$ larger in the Cooling condition $\left(\Delta T_{\text {chest }}=-1.465 \pm 0.14\right.$ $\left.{ }^{\circ} \mathrm{C}\right)$ than the Control condition $\left(\Delta \mathrm{T}_{\text {chest }}=-0.21 \pm 0.14^{\circ} \mathrm{C}\right)$.

The HR data from intermittent cooling during exercise resulted in almost identical responses for both conditions (Figure 6). For HR during rest breaks, we found no significant difference $\left(F_{1,18}=0.308, p=0.586\right.$, partial $\left.\eta^{2}=0.017,1-\beta=0.082\right)$ between the Cooling condition $(\mathrm{HR}=147.6 \pm 2.6 \mathrm{bpm})$ and the Control condition $(\mathrm{HR}=150.1 \pm 3.5 \mathrm{bpm})$; however we did find significant differences between pre- and post-test $\left(F_{5,90}=429.796, p<0.001\right)$. For HR during recovery, we found no significant $\left(F_{1,18}=1.552, p=0.229\right.$, partial $\left.\eta^{2}=0.079,1-\beta=0.219\right)$ differences between conditions; however we did find significant differences between pre- and post-test $\left(F_{5,90}=21.919, p<0.001\right)$.

\section{Perceptual Responses}

The thermal responses (Figure 7$)$ were significantly $\left(F_{1,18}=43.038, p=0.026\right)$ lower for 332 the Cooling condition ( $4.4 \pm 0.2$ points) compared to the Control condition (5.0 \pm 0.2 points).

333 The thirst responses (Figure 8) approached significance $\left(F_{1,18}=5.038, p=0.051\right.$, partial $\eta^{2}=$ $3340.359,1-\beta=0.517)$ with thirst response for the Cooling condition being lower $(4.5 \pm 0.3$ points) 335 than the Control condition (5.3 \pm 0.4 points). The RPE responses (Figure 9) were not 336 significantly $\left(F_{1,18}=2.291, p=0.164\right.$, partial $\left.\eta^{2}=0.203,1-\beta=0.273\right)$ different between conditions. 
No other significant differences were found. No symptoms of exertional heat illness using the ESQ were found for either group. No significant correlations (Table 3) were found between thermoregulatory, cardiovascular, and perceptual responses at the end of exercise for Control condition or the Cooling condition.

\section{Hydration Status}

Hydration status (Table 4) was not significantly $\left(F_{1,9}=.008, p=0.993\right.$, partial $\eta^{2}=0.001,1$ $\beta=0.051)$ different between conditions. However, both conditions became significantly hypohydrated during the exercise session with a mean \% body mass loss $=-2.2 \pm 0.8 \%\left(F_{1,9}\right.$ $=182.637, p<0.001)$. The urinalysis data revealed no significant differences between conditions $\left(U_{\text {col }} F_{1,9}=2.548, p=.145\right.$, partial $\eta^{2}=0.221,1-\beta=0.298 ; U_{s g} F_{1,9}=1.514, p=.250$, partial $\eta^{2}=$ $0.144,1-\beta=0.197)$; however, both $U_{c o l}$ and $U_{s g}$ indicated significant increased post-exercise. $\mathrm{U}_{\text {col }}$ for the both conditions was significantly $\left(\mathrm{F}_{1,9}=56.077, p<0.001\right)$ increased $33.3 \%$ postexercise $(6.8 \pm 0.2)$ compared to pre-exercise $(4.5 \pm 0.4)$. $U_{s g}$ for the both groups was significantly $\left(F_{1,9}=17.612, p=0.002\right)$ increased $33.3 \%$ post-exercise $(1.022 \pm 0.002)$ compared to pre-exercise (1.019 \pm 0.002$)$. No significant differences between conditions were found for $F_{\mathrm{vol}}(p$ $=0.250)$ or sweat rate $(p=0.961)$.

\section{Environmental Conditions}

Environmental conditions measured in the sun and in the shade are demonstrated in Figure 2. Outdoor environmental conditions were significantly higher $\left(t_{20}=4.99, p<.001\right)$ in the sun $\left(\mathrm{WBGT}_{0}=27.1 \pm 0.8^{\circ} \mathrm{C}\right)$ than shade $\left(\mathrm{WBGT}_{0}=25.4 \pm 0.8^{\circ} \mathrm{C}\right)$.

\section{DISCUSSION}

\section{Thermoregulatory and Cardiovascular Responses}

The most important findings of this investigation were that although we found 367 significantly decreased thermoregulatory response in the superficial cooling condition $\left(T_{\mathrm{gi}}=\right.$ 
4368

$38.2 \pm 0.2^{\circ} \mathrm{C}$ vs $\mathrm{T}_{\mathrm{gi}}=38.5 \pm 0.1^{\circ} \mathrm{C}$ for cooling and control, respectively), these decreases were small and not clinically important. Furthermore, overall cooling rates were not significantly different between the cooling and control conditions, indicating that the superficial cooling vest was not effective in reducing the core body temperature increase during intense exercise in the heat. Finally, although we elected to evaluate intermittent cooling followed by continuous cooling during recovery, most applications of this type of "phase change" cooling vest would most likely be during recovery from exercise only. We found no significant differences between cooling and control during 30 min of recovery from intense exercise in the heat. This finding implies that recovery by sitting in the shade and rehydrating versus using a superficial cooling vest are equally effective in reducing core body temperature after intense exercise in the heat.

We found that the core body temperature of individuals exercising in the heat remained elevated despite efforts of intermittent, superficial cooling (Figure 4). In agreement with previous investigations $(13,25,33)$ superficial cooling vests were not effective in lowering $T_{g i}$; however, use of the cooling vest did attenuate the rise of $T_{g i}$ during intense exercise in the heat. 382 Furthermore, cooling rate for the current investigation was similar to the current researchers' 383 previous investigation (vest group $=0.03 \pm 0.01^{\circ} \mathrm{C} \cdot \mathrm{min}^{-1}$, no-vest group $=0.03 \pm 0.01^{\circ} \mathrm{C} \cdot \mathrm{min}^{-1}$ ) with 384 a similar 30 min recovery period.(25) These cooling rates are far inferior to the plethora of 385 evidence relating to cooling rates of hyperthermic individuals during cold-water immersion which 386 are consistently reported to be $0.16-0.2^{\circ} \mathrm{C} \cdot \mathrm{min}^{-1}\left(0.29-0.36^{\circ} \mathrm{F} \cdot \mathrm{min}^{-1}\right)$ or upward of $0.35^{\circ} \mathrm{C} \cdot \mathrm{min}^{-1}$ $387\left(0.63^{\circ} \mathrm{F} \cdot \mathrm{min}^{-1}\right)$ when multiple elements that influence cooling rates are in place.(9) Similar to our findings- other studies found significant decreases in the rate of rise of $T_{g i}$ with intermittent cooling during exercise in the heat. $(5,28)$ Price $(28)$ evaluated the physiological effects of intermittent, superficial cooling on exercise in the heat during a simulated soccer match. A significant decrease in $\mathrm{T}_{\text {gi }}$ was found between the first and second bouts of exercise in 392 the pre- plus mid-cooling condition. Similarly, Barr(5) found that in firefighters $T_{g i}$ and thermal 393 sensations were significantly lower during the second bout of exercise when wearing a cooling 
vest in between two exercise bouts. Both studies $(5,28)$ ' provided participants a 15 min rest break between exercise bouts. The differences in time between the 15 min rest breaks compared to the 5 min rest breaks in the current investigation likely explains why $T_{\text {gi }}$ decreased significantly compared to the control condition. In addition, in the present study during recovery, the overall decrease in $T_{g i}$ was larger for the Control condition because the final temperatures were higher in the Control condition providing a larger temperature gradient for cooling. Although our experimental protocol was based on the NATA recommendations of 5-min rest breaks every 20 min of exercise in Red Flag conditions (6), longer rest breaks may be required to achieve sufficient cooling to reduce elevated core body temperature and prevent dangerous hyperthermia from developing.

Superficial cooling applications vary considerably in concept, design, and application.

Cadarette (8) studied the effects of constant cooling versus intermittent cooling versus no cooling in military personnel wearing chemical protective clothing during continuous treadmill walking $80 \mathrm{~min}$. The protective uniform consisted of a liquid cooling garment with cold water circulating either continuously, intermittently ( $2 \mathrm{~min}$ on and $2 \mathrm{~min}$ off), or not at all. This researcher found that continuous and intermittent cooling during exercise was effective in lowering $T_{\text {gi. }}$ (8) These applications of superficial cooling may be effective in soccer games,

411 firefighter training, and military convoys where longer breaks or seated activities allow 412 continuous cooling. However, when applying these findings to generalized athletic settings 413 such as football practices and games the rest periods are much shorter and the equipment 414 required continuous cooling is impossible in these settings.

$415 \quad$ Heart rate responses (Figure 6) were not affected by the superficial cooling indicating 416 that the cardiovascular strain experienced by the participants was not attenuated with the 417 cooling garment. Rest in the shade and rehydration was equally effective in reducing 418 cardiovascular strain. Armstrong (4) reported that in football athletes, cardiovascular strain was 419 increased in individuals wearing a full football uniform compared to control. However, cessation 
420 of exercise reduced heart rate regardless of the equipment configuration. Clearly, during 421 exercise in hot, humid environments, removing equipment, resting in the shade or air 422 conditioned environment, and rehydration are effective strategies in reducing thermoregulatory and cardiovascular strain. The use of superficial cooling garments may attenuate the rise in core body temperature during exercise in the heat, but these garments are not particularly useful in accelerating core body cooling or reducing cardiovascular strain during exercise in the heat. Proper precautions must be followed when athletes are intensely exercising in the heat. Using superficial cooling garments intermittently during exercise may provide a small measure of protection but seated rest and rehydration in the shade at 15-20 min intervals is essential to avoid dehydration, hyperthermia, and exertional heat illness.

Hyperthermia is common among athletes and occurs in a variety of environments and athletic settings and when combined with dehydration and heat stress can lead to exertional heat illness. Utilizing effective cooling modalities combined with rest and rehydration may be 433 useful in preventing dangerous hyperthermia from developing. The effectiveness of a variety of 434 cooling methods have been evaluated in individuals after exercise including cold water 435 immersion, shade, cold fans, vests, hoods, ice buckets, and ice towels.(13) In athletes 436 experiencing mild hyperthermia, cold water immersion and ice buckets have consistently 437 resulted in a significantly greater decrease in core body temperature. While these cooling 438 strategies are recommended for the treatment of $\mathrm{EHI},(13)$ these cooling strategies are 439 impractical for use in decreasing core body temperature during brief recovery periods between exercise bouts. Although this investigation was not about exercise performance, we found an 441 extensive systematic literature review (29) reporting that aerobic exercise performance can be 442 enhanced with the use of cooling modalities. In this review, cold water immersion, cooling 443 vests, cooling collars, and hand cooling devices all provided subjects with some exercise 444 benefits. Many of these modalities could be used during athletic events such as between tennis 445 matches or during halftime of other outdoor events such as American football or soccer.(29) 
446 Some specific cooling modalities may provide a benefit to performance when used before and in

between exercise bouts; however, in cases of dangerous hyperthermia cold-water immersion is the modality with the highest cooling rate.

\section{Perceptual Responses}

Thermal Sensations. The important findings of this investigation were that thermal sensation was significantly lower for the Cooling condition, indicating that superficial cooling was effective in reducing participants' sensation of heat. This finding is largely explained in that the cooling vest was effective in significantly lowering $T_{\text {chest. }}$ Although $T_{\text {gi }}$ was significantly different 456 between conditions, we found that at the end of break 1 and 2 the Cooling condition produced a 457 higher $\mathrm{T}_{\mathrm{gi}}$ than the Control condition- indicating that rest in the shade alone is just as effective in reducing thermal strain as wearing the cooling vest. These findings are similar to previous investigations.(8)'(28) When superficial blood is cooled, it is returned to the core of the body to reduce the temperature of the core; however, the cooling vest was not effective in blunting the elevation of $T_{\text {gi }}$ which continued to increase in both conditions throughout exercise as a result of the exercise intensity and heat stress.

Participants' thermal perceptions during the Cooling condition were very intense coldness initially; however, after 5-min of cooling the participants' reported that the vest felt "like a wet t-shirt" and that it had lost much of its cooling capacity. Skin sensations are known to adapt over time, so it is not clear if the vest material decreased in temperature or if the participants' cognitive perception of coldness had accommodated. According to Herrera (22) ice massage, ice pack, and cold water immersion reduced sensory nerve conduction velocity. Thermal sensation sensitivity may have decreased after 5 min of cold application, potentially explaining why subjects commented on the change in temperature of the cooling vest. Coaches and athletic trainers should exercise caution when athletes are using superficial cooling vests during intense exercise in the heat as the vest imparts a sensation of coolness on the skin while 
the core body temperature is not reduced. This finding is important as athletes using intermittent cooling may continue to exercise intensely without pacing themselves and potentially develop dangerous hyperthermia.

Thirst Sensations. Although both conditions resulted in hypohydration (2.2\%), thirst sensations were slightly lower in the Cooling condition $(p=0.051)$ than the Control condition. Thirst sensations may have been less in the Cooling condition perhaps because as exercise progressed, participants in the Cooling condition were less able to perceive hypohydration developing from the intense exercise and heat stress. According to Maresh (26) thirst may be an underlying cue for increased RPE during exercise in a hot environment. In the current investigation, no correlations were found with thirst and RPE at the end of exercise; however, as expected RPE and thirst decreased following each rest break independent of superficial cooling. Furthermore, thirst did increase progressively at the end of each exercise bout throughout the investigation with the highest thirst rating at the end of the last exercise bout, regardless of condition. As the exercise task progresses, exercise tolerance may be related to perceptions of thirst, potentially negatively impacting the perception of exercise intensity. Lower thirst sensation in the cooling condition is an important practical finding because subjects consumed less fluid and became dehydrated. Superficial cooling may make athletes feel cooler and therefore neglect to consume enough fluid to avoid dehydration and exertional heat illness.

Rating of Perceived Exertion. Cooling vest companies commonly advertise anecdotal 492 evidence that superficial cooling can enhance performance because the subject feels cooler 493 and is therefore less fatigued. We found that when participants wore the cooling vests they 494 informed investigators that it made them feel "re-energized", "refreshed", and "cold and ready to 495 go". However, when this evidence was transferred into a quantifiable measurement- RPE, there 496 were no significant differences between conditions. This finding indicates that exercise caused 497 an increase in RPE regardless of cooling and that rest- independent of cooling, decreased RPE. Indeed, in the Cooling condition we found no significant correlation between the RPE and 
499 thermal sensations, indicating that rest alone reduced RPE. This finding indicates that the 500 cooling vest altered thermal perceptions of heat without affecting RPE or cardiovascular

responses. Similar to Johnson's (24) findings, the perceptual scales indicated that participants found it difficult to perceptually rate exercise conditions and exercise strain as hyperthermia developed. Coaches should be aware that intermittent superficial cooling during exercise does not appreciably decrease perceptions of exertion. Athletes exercising intensely in the heat, regardless of using a superficial cooling garment, must take regular rest and rehydration breaks to avoid dangerous hyperthermia.

Limitations of this Investigation. A limitation of field research in general is that environmental conditions cannot be tightly controlled without an environmental chamber. However, we collected data at the same time each day for 11 days over a 3-week period in JulyAugust in a tropical climate with minor variations in temperature (Figure 2). Further, we assert that field research is more generalizable than laboratory research as it occurs in the actual 512 conditions that American football players would be training and conditioning. Another limitation 513 of this investigation was that the effects of intermittent, superficial cooling on exercise 514 performance was not examined. Exercise performance was not a primary objective of our 515 investigation and many previous investigations, including a very detailed and thorough 516 systematic literature review (29) have examined the effects of superficial cooling on exercise 517 performance. One previous study (15) examined the effects of applying a cooling vest before 518 exercise to determine a change in core body temperature; however no significant results were 519 found. In addition, limited evidence (14) exists to support the concept of self-pacing and the 520 limitations of a critical internal temperature. In terms of performance, there is strong evidence in 521 the literature that some specific cooling modalities may provide a benefit to performance when 522 used before and in between exercise bouts.(29) Therefore, future studies should be designed 523 to determine the relationship between intermittent, superficial cooling, with how individuals perceive the skin coolness and modify behavior or exercise pacing. 
525

526

527

528

\section{PRACTICAL APPLICATION}

Superficial cooling garments are a popular way for athletes to feel cooler while exercising in hot environments. These garments have been recently marketed to athletic trainers, athletes, and coaches as a means to rapidly reduce core body temperature and presumably for the prevention and treatment of EHI. The findings of this investigation were: 1.) although statistically significant- cooling did not sufficiently attenuate $T_{g i}$ to be clinically relevant; 2) intermittent cooling did it decrease thirst, RPE, or HR; 3.) although participants drank water ad libitium during rest breaks- they became mildly dehydrated with the cooling condition slightly more dehydrated (not significant) than the control condition; 4) in an athletic setting, intermittent, superficial cooling was effective in reducing thermal sensations and $\mathrm{T}_{\text {chest }}$; and 5 .) rest in the shade and rehydration was similarly effective to the superficial cooling garment in reducing core body temperature during and after exercise. In conclusion, since athletes may feel cooler but their core body temperature is still elvated-perceptual responses should be carefully considered when deciding to use cooling vests during extremely hot conditions. Athletes may feel cooler, but they may not be able to perceive the increase in core body temperature making them potentially at further risk for EHI.

Coaches, athletic trainers, and strength and conditioning specialists, should understand that cooling vests are not to be used for treatment of exertional heat illness, However, these garments may be effective in reducing thermal sensations. Superficial cooling vests may be helpful in preventing heat illness or may be used in adjunct with full body ice water immersion to treat EHI.(9) It is important to remember that when athletes are exercising in the heat 5-10 min rest and rehydration breaks must occur in the shade every 15-20 min, minimal equipment should be worn, and individuals at risk should not participate. Should dangerous hyperthermia occur $\left(\mathrm{T}_{\mathrm{gi}}>40^{\circ} \mathrm{C}\right)$ coaches should summon medical help and immediately begin the recommended "gold standard" treatment for rapidly cooling hyperthermic athletes-full body ice 
552 water immersion. $(1,9)$

6

7

8 


\section{ACKNOWLEDGMENTS}

7554

855

9 556 11

We are grateful that Polar Products, Inc. (Stow, $\mathrm{OH}$ ) provided the cooling vests for this investigation. The investigators would like to thank the participants for their time and efforts as well as the Kinesiology and Rehabilitation Science students who helped collect data for this investigation, especially Melissa Shrum who made an important contribution to our study. The 559 authors have no conflict of interest and we have no professional relationships with companies or 560 manufacturers who will benefit from the results of the present study. There was no specific 561 grant support for the study. The results of the present study do not constitute endorsement of 562 the product by the authors or the NSCA. 


\section{LIST OF TABLES}

Table 1. Protocol for warm-up, anaerobic exercise, and recovery periods

Table 2. Correlation $(\mathrm{R})$ and significance $(\mathrm{P})$ for thermoregulatory, cardiovascular, and perceptual responses for cooling and control conditions at the beginning of recovery $(n=10)$ No significant relationships were found.

Note. $\mathrm{RPE}=$ rating of perceived exertion, $\mathrm{T}_{\mathrm{gi}}=$ core body temperature, $\mathrm{T}_{\text {chest }}=$ skin temperature, and $\mathrm{HR}=$ heart rate

Table 3. Hydration status pre-exercise, post-exercise, and the difference (delta) ( $\mathrm{n}=10$ mean $\pm \mathrm{SD}$ )

${ }^{*}$ Both groups became significantly hypohydrated during the exercise session $(p \leq 0.001)$. No other significant differences were found. 
583

584

585

586

587

10588

11589

12590

13591

14592

15593

16594

18595

19596

20597

21598

22599

23600

24601

\section{LIST OF FIGURES}

Figure 1. Research design

Figure 2. Environmental conditions in the sun and in the shade (11 days)

$\mathrm{WBGT}_{0}=$ wet bulb globe temperature outdoors

*Significantly different than Shade $(p<0.05)$

Figure 3. Polar Products Cool58 ${ }^{\mathrm{TM}}$ Phase Change Vest.

Retrieved from:

http://www.polarproducts.com/polarshop/pc/Cool58-Phase-Change-Zipper-Vest-16p197.htm. Accessed on April 20, 2013.

Figure 4. Core temperature for intermittent cooling during exercise and recovery $(n=10$, mean \pm SD)

During exercise we found no significant differences between conditions ( $p=.674$ ); however we did find a significant decrease in $\mathrm{T}_{\text {gi }}$ during Break 3 (pre-test $=38.50 \pm .13^{\circ} \mathrm{C}$ vs. post-test $=$ $38.18 \pm .13^{\circ} \mathrm{C}$ ) for both conditions. For $\mathrm{T}_{\mathrm{gi}}$ during recovery, we found significant differences between condition $(p=.452)$ and $T_{\text {gi }}$ progressively decreased during recovery $(p<.001)$. For the $\Delta \mathrm{T}_{\mathrm{gi}}$ we found no significant differences for condition $(p=.152)$.

Figure 5. Skin (chest) temperature for intermittent cooling during exercise and recovery $(n=10$, mean \pm SD)

${ }^{*}$ During exercise we found significantly $(p<0.001)$ decreased skin temperature for the Cooling condition $\left(T_{\text {chest }}=31.85 \pm 0.43^{\circ} \mathrm{C}\right)$ compared to the Control condition $\left(T_{\text {chest }}=34.38 \pm 0.43^{\circ} \mathrm{C}\right)$. During recovery, we found significantly $(p<0.001)$ lower skin temperature in the Cooling condition $\left(T_{\text {chest }}=31 \cdot 24 \pm 0.47^{\circ} \mathrm{C}\right)$ compared to the Control condition $\left(T_{\text {chest }}=33.48 \pm 0.47^{\circ} \mathrm{C}\right)$.

Figure 6. Heart rate for intermittent cooling during exercise and recovery $(n=10$, mean $\pm S D)$ During exercise we found no significant difference $(p=0.586)$ between the Cooling condition $(\mathrm{HR}=147.6 \pm 2.6 \mathrm{bpm})$ and the Control condition $(\mathrm{HR}=150.1 \pm 3.5 \mathrm{bpm})$; however we did find *significant differences between pre- and post-test $(p<0.001)$. During recovery, we found no significant $(p=0.229)$ differences between conditions; however we did find *significant differences between pre- and post-test $(p<0.001)$.

Figure 7. Thermal responses for intermittent cooling during exercise and recovery $(n=10$, mean \pm SD)

${ }^{*}$ Significantly $\left(F_{1,18}=43.038, p=0.026\right)$ lower for the Cooling condition ( $4.4 \pm 0.2$ points) compared to the Control condition (5.0 \pm 0.2 points).

Figure 8. Thirst responses for intermittent cooling during exercise and recovery $(n=10$, mean \pm SD)

Approached significance $(p=0.051)$ with the Cooling condition lower $(4.467 \pm 0.288$ points) than the Control condition (5.267 \pm 0.392 points).

Figure 9. RPE responses for intermittent cooling during exercise and recovery $(n=10$, mean $\pm S D$ )

No significant $(p=0.164)$ differences between conditions. 


\section{REFERENCES}

1. Armstrong LE, Crago AE, Adams R, Roberts WO, Maresh CM. Whole-body cooling of hyperthermic runners: comparison of two field therapies. Am J Emerg Med. 1996;14(4):355-358.

2. Armstrong LE, Soto JA, Hacker FT, Jr., Casa DJ, Kavouras SA, Maresh CM. Urinary indices during dehydration, exercise, and rehydration. Int J Sport Nutr. 1998;8(4):345-355.

3. Armstrong LE, Casa DJ, Millard-Stafford M, Moran DS, Pyne SW, Roberts WO. American College of Sports Medicine position stand. Exertional heat illness during training and competition. Med Sci Sports Exerc. 2007;39(3):556-572.

4. Armstrong LE, Johnson EC, Casa DJ, et al. The American football uniform: uncompensable heat stress and hyperthermic exhaustion. J Athl Train. 2010;45(2):117127.

5. Barr D, Gregson W, Sutton L, Reilly T. A practical cooling strategy for reducing the physiological strain associated with firefighting activity in the heat. Ergonomics. 2009;52(4):413-420.

6. Binkley HM, Beckett J, Casa DJ, Kleiner DM, Plummer PE. National Athletic Trainers' Association Position Statement: Exertional Heat Illnesses. J Athl Train. 2002;37(3):329343.

7. Borg G. Perceived exertion as an indicator of somatic stress. Scand J Rehabil Med. 1970;2(2):92-98.

8. Cadarette BS, Cheuvront SN, Kolka MA, Stephenson LA, Montain SJ, Sawka MN. Intermittent microclimate cooling during exercise-heat stress in US army chemical protective clothing. Ergonomics. 2006;49(2):209-219.

9. Casa DJ, McDermott BP, Lee EC, Yeargin SW, Armstrong LE, Maresh CM. Cold water immersion: the gold standard for exertional heatstroke treatment. Exerc Sport Sci Rev. 2007;35(3):141-149.

10. Cleary MA, Hetzler RK, Wages JJ, Lentz MA, Stickley CD, Kimura IF. Comparisons of age-predicted maximum heart rate equations in college-aged subjects. J Strength Cond Res. 2011;25(9):2591-2597.

11. Cooper ER, Ferrara MS, Broglio SP. Exertional heat illness and environmental conditions during a single football season in the southeast. J Athl Train. 2006;41(3):332-336.

12. Database NCAALS. 17.9.2.3 Five-Day Acclimatization Period and 17.9.2.4 Preseason Activities After Five-Day Acclimatization Period. Retreived from: https://web1.ncaa.org/LSDBi/exec/homepage. Accessed on: September 17, 2011.

13. Demartini JK, Ranalli GF, Casa DJ, et al. Comparison of body cooling methods on physiological and perceptual measures of mildly hyperthermic athletes. J Strength Cond Res. 2011;25(8):2065-2074.

14. Duffield R, Green R, Castle P, Maxwell N. Precooling can prevent the reduction of selfpaced exercise intensity in the heat. Med Sci Sports Exerc. 2010;42(3):577-584.

15. Duffield R, Dawson B, Bishop D, Fitzsimons M, Lawrence S. Effect of wearing an ice cooling jacket on repeat sprint performance in warm/humid conditions. Br J Sports Med. 2003;37(2):164-169.

16. Engell DB, Maller O, Sawka MN, Francesconi RN, Drolet L, Young AJ. Thirst and fluid intake following graded hypohydration levels in humans. Physiol Behav. 1987;40(2):229236.

17. Gao C, Kuklane K, Holmer I. Cooling vests with phase change material packs: the effects of temperature gradient, mass and covering area. Ergonomics. 2010;53(5):716-723.

18. Gao C, Kuklane K, Holmer I. Cooling vests with phase change materials: the effects of melting temperature on heat strain alleviation in an extremely hot environment. Eur J Appl Physiol. 2011;111(6):1207-1216. 
19. Gellish RL, Goslin BR, Olson RE, McDonald A, Russi GD, Moudgil VK. Longitudinal modeling of the relationship between age and maximal heart rate. Med Sci Sports Exerc. 2007;39(5):822-829.

20. Gonzalez-Alonso J. Hyperthermia impairs brain, heart and muscle function in exercising humans. Sports Med. 2007;37(4-5):371-373.

21. Hasegawa H, Takatori T, Komura T, Yamasaki M. Wearing a cooling jacket during exercise reduces thermal strain and improves endurance exercise performance in a warm environment. J Strength Cond Res. 2005;19(1):122-128.

22. Herrera E, Sandoval MC, Camargo DM, Salvini TF. Motor and sensory nerve conduction are affected differently by ice pack, ice massage, and cold water immersion. Phys Ther. 2010;90(4):581-591.

23. Hornery DJ PS, Mujika I, Hahn A. Physiological and performance benefits of halftime cooling. 2005 8(1):15-25.

24. Johnson EC, Ganio MS, Lee EC, et al. Perceptual responses while wearing an American football uniform in the heat. J Athl Train. 2010;45(2):107-116.

25. Lopez RM, Cleary MA, Jones LC, Zuri RE. Thermoregulatory influence of a cooling vest on hyperthermic athletes. J Athl Train. 2008;43(1):55-61.

26. Maresh CM, Gabaree-Boulant CL, Armstrong LE, et al. Effect of hydration status on thirst, drinking, and related hormonal responses during low-intensity exercise in the heat. $J$ Appl Physiol. 2004;97(1):39-44.

27. Mitchell JB, Schiller ER, Miller JR, Dugas JP. The influence of different external cooling methods on thermoregulatory responses before and after intense intermittent exercise in the heat. J Strength Cond Res. 2001;15(2):247-254.

28. Price MJ, Boyd C, Goosey-Tolfrey VL. The physiological effects of pre-event and midevent cooling during intermittent running in the heat in elite female soccer players. Appl Physiol Nutr Metab. 2009;34(5):942-949.

29. Ranalli GF, Demartini JK, Casa DJ, McDermott BP, Armstrong LE, Maresh CM. Effect of body cooling on subsequent aerobic and anaerobic exercise performance: a systematic review. J Strength Cond Res. 2010;24(12):3488-3496.

30. Reilly T, Drust B, Gregson W. Thermoregulation in elite athletes. Curr Opin Clin Nutr Metab Care. 2006;9(6):666-671.

31. Sampson JB, Kobrick JL. The environmental symptoms questionnaire: revisions and new filed data. Aviat Space Environ Med. 1980;51(9 Pt 1):872-877.

32. Webster J, Holland EJ, Sleivert G, Laing RM, Niven BE. A light-weight cooling vest enhances performance of athletes in the heat. Ergonomics. 2005;48(7):821-837.

33. Young AJ, Sawka MN, Epstein Y, Decristofano B, Pandolf KB. Cooling different body surfaces during upper and lower body exercise. J Appl Physiol. 1987;63(3):1218-1223.

34. Young AJ SM, Epstein Y, Decristofano B, Pandolf KB. Cooling different body surfaces during upper and lower body exercise. 1987;63(3 ):1218-1223. 
Table 1. Protocol for warm-up, anaerobic exercise, and recovery periods

\begin{tabular}{|c|c|c|}
\hline $\begin{array}{l}\text { Running } \\
\text { Time }\end{array}$ & $\begin{array}{l}\text { Segment } \\
\text { Time }\end{array}$ & Exercise on standard football field \\
\hline & & Warm-Up (10 min) \\
\hline $0 \min$ & $10 \min$ & $\begin{array}{l}\text { Dynamic warm-up exercises to the } 15 \text { yd line and back: jog, skip, } \\
\text { side shuffle, single leg knee hugs with hip external rotation, back pedal, } \\
\text { butt kick, lateral bound, Spiderman walk, power skip for height, front } \\
\text { squats, side lunges, carioca, high knees, quad stretch with calf raise, } \\
\text { march walk, toe kick, jog, stride, sprint. }\end{array}$ \\
\hline \multirow[t]{2}{*}{$10 \mathrm{~min}$} & $5 \mathrm{~min}$ & Rest with additional stretches, as needed, collect data post-warm-up \\
\hline & & Exercise Protocol 1 (20 min) \\
\hline $15 \min$ & $2 \min$ & Medicine ball toss with 10 squats in place, and repeat for $100 \mathrm{yd}$. \\
\hline $17 \min$ & $2 \min$ & $\begin{array}{l}\text { End zone workout 1: } 10 \text { push-ups, } 10 \text { mountain climbers, lunges } 10 \\
\text { yd, lateral lunges } 10 y d \text {, pass medicine ball around the waist } 10 \mathrm{x} \text {. }\end{array}$ \\
\hline $19 \min$ & $2 \min$ & Medicine ball toss same as above \\
\hline $21 \min$ & $2 \min$ & End zone workout 1 same as above \\
\hline $23 \mathrm{~min}$ & $1.5 \mathrm{~min}$ & Standing rest \\
\hline $24.5 \min$ & $2.5 \mathrm{~min}$ & Agility series: run $5 \mathrm{yd}$ left, run $10 \mathrm{yd}$ right, back to start (20 s rest) $5 \mathrm{x}$ \\
\hline $27 \mathrm{~min}$ & $1 \mathrm{~min}$ & Standing rest \\
\hline $28 \mathrm{~min}$ & $7 \mathrm{~min}$ & $\begin{array}{l}\text { Jog/stride/sprint: } 30 \text { s slow jog, } 20 \text { s stride, } 10 \text { s full sprint ( } 1 \text { min } \\
\text { break), repeat. }\end{array}$ \\
\hline \multirow[t]{2}{*}{$35 \mathrm{~min}$} & $5 \mathrm{~min}$ & Rest Break 1 in shade: Collect data pre- and post- \\
\hline & & Exercise Protocol 2 (20 min) \\
\hline $40 \min$ & $5 \mathrm{~min}$ & $\begin{array}{l}\text { Pass routes: } 1^{\text {st }} \text { set: Sprint } 2 \times 10 y d, 2 \times 15 y d, 1 \times 20 y d ; 2^{\text {nd }} \text { set: } 1 \times \\
20 y d, 2 \times 15 \text { yd, } 2 \times 10 \text { yd. Slow jog back to the starting line ( } 20 \text { s rest). }\end{array}$ \\
\hline $45 \min$ & $1 \mathrm{~min}$ & Standing rest \\
\hline $46 \min$ & $6 \mathrm{~min}$ & $\begin{array}{l}\text { End zone workout 2: jog } 20 \mathrm{~s} \text {, jump forwards/backwards } 20 \mathrm{~s} \text {, jogging } \\
20 \mathrm{~s} \text {, jump side to side } 20 \mathrm{~s} \text {, jog } 20 \mathrm{~s} \text {, jump for height } 20 \mathrm{~s} \text {, jog } 20 \mathrm{~s} 2 \mathrm{x}\end{array}$ \\
\hline $52 \min$ & $1 \mathrm{~min}$ & Standing rest \\
\hline $53 \mathrm{~min}$ & $2 \min$ & 40-yd dash: sprint $40 \mathrm{yd}$ (20 s rest) $4 \mathrm{x}$ \\
\hline $55 \mathrm{~min}$ & $1 \mathrm{~min}$ & Standing rest \\
\hline $56 \min$ & $\begin{array}{l}3 \min +1 \\
\min \text { rest }\end{array}$ & $\begin{array}{l}60 \text { yd shuttle run: run } 5 \text { yd and back, } 10 \text { yd and back, } 15 \text { yd and back } \\
\text { (20 s rest) } 4 x\end{array}$ \\
\hline \multirow[t]{2}{*}{$60 \mathrm{~min}$} & $5 \mathrm{~min}$ & Rest Break 2 in shade: Collect data pre- and post- \\
\hline & & Exercise Protocol 3 (20 min) \\
\hline $65 \min$ & $4 \min$ & $\begin{array}{l}\text { Build up running: run } 30 \text { yd at } 50 \% \text { effort, run } 30 \text { yd at } 70 \% \text { effort, run } \\
20 \text { yd at } 80 \% \text { effort, sprint } 20 \text { yd at } 100 \% \text { effort ( } 1 \text { min rest) } 4 x\end{array}$ \\
\hline $69 \min$ & $1 \mathrm{~min}$ & Standing rest \\
\hline $70 \mathrm{~min}$ & $6 \mathrm{~min}$ & $\begin{array}{l}\text { End zone Workout 2: jog } 20 \mathrm{~s} \text {, jump forward/backward } 20 \mathrm{~s} \text {, jog } 20 \mathrm{~s} \text {, } \\
\text { jump side-to-side } 20 \mathrm{~s} \text {, jog } 20 \mathrm{~s} \text {, jump up } 20 \mathrm{~s} \text {, jog } 20 \mathrm{~s} .2 \mathrm{x}\end{array}$ \\
\hline $76 \min$ & $1 \mathrm{~min}$ & Standing rest \\
\hline $77 \min$ & $3 \mathrm{~min}$ & $\begin{array}{l}\text { Power series: } 10 \text { jump tucks, } 10 \text { vertical lunges, } 15 \text { lateral ice skaters, } \\
\text { bound } 20 \text { yd, standing broad jumps } 20 \text { yd. } 2 \text { x }\end{array}$ \\
\hline $80 \mathrm{~min}$ & $1 \mathrm{~min}$ & Standing rest \\
\hline $81 \mathrm{~min}$ & $4 \mathrm{~min}$ & Jog/stride/sprint: same as above. \\
\hline \multirow[t]{2}{*}{$85 \mathrm{~min}$} & $5 \mathrm{~min}$ & Rest Break 3 in shade: Collect data pre- and post- \\
\hline & & Recovery Period (30 min) \\
\hline $90 \min$ & $30 \mathrm{~min}$ & Recovery Period: Collect data every 5 min \\
\hline
\end{tabular}


Table 2. Environmental Conditions in the Sun and in the Shade (10 days)

\begin{tabular}{|c|c|c|c|c|c|}
\hline \multicolumn{6}{|c|}{ Sun } \\
\hline & $\begin{array}{c}\text { WBGT }^{*} \\
\left({ }^{\circ} \mathbf{C}\right)\end{array}$ & $\begin{array}{c}\text { Dry Bulb } \\
\left({ }^{\circ} \mathrm{C}\right)\end{array}$ & $\begin{array}{c}\text { Relative } \\
\text { Humidity* } \\
(\%)\end{array}$ & $\begin{array}{c}\text { Turf } \\
\text { Temperature* } \\
\left({ }^{\circ} \mathrm{C}\right)\end{array}$ & $\begin{array}{c}\text { Turf Relative } \\
\text { Humidity }^{\star} \\
(\%)\end{array}$ \\
\hline Mean & 27.0 & 29.0 & 44.2 & 41.0 & 32.4 \\
\hline SD & 0.8 & 1.0 & 6.5 & 4.4 & 7.6 \\
\hline Min & 25.8 & 27.2 & 36.0 & 35.4 & 23.8 \\
\hline Max & 28.1 & 30.4 & 57.0 & 50.8 & 50.0 \\
\hline \multicolumn{6}{|c|}{ Shade } \\
\hline & $\begin{array}{c}\text { WBGT } \\
\left({ }^{\circ} \mathrm{C}\right)\end{array}$ & $\begin{array}{c}\text { Dry Bulb } \\
\left({ }^{\circ} \mathbf{C}\right)\end{array}$ & $\begin{array}{c}\text { Relative } \\
\text { Humidity } \\
(\%)\end{array}$ & $\begin{array}{c}\text { Turf } \\
\text { Temperature } \\
\left({ }^{\circ} \mathrm{C}\right)\end{array}$ & $\begin{array}{c}\text { Turf Relative } \\
\text { Humidity } \\
(\%)\end{array}$ \\
\hline Mean & 25.4 & 28.7 & 50.8 & 32.8 & 48.4 \\
\hline SD & 0.9 & 1.0 & 6.0 & 2.8 & 10.9 \\
\hline Min & 24.3 & 26.6 & 43.0 & 28.7 & 34.1 \\
\hline Max & 26.8 & 29.7 & 64.0 & 38.3 & 68.4 \\
\hline
\end{tabular}

WBGTo = wet bulb globe temperature outdoors

${ }^{*}$ All environmental conditions (except Dry Bulb) were significantly higher in the Sun than Shade $(p<0.05)$ 
Table 3. Correlation $(R)$ and significance $(P)$ for thermoregulatory, cardiovascular, and perceptual responses for Cooling and Control conditions at the beginning of recovery $(n=10)$

Thermal

\begin{tabular}{ccccccccccccc}
\hline & \multicolumn{2}{c}{ Control } & \multicolumn{2}{c}{ Cooling } & \multicolumn{2}{c}{ Control } & \multicolumn{2}{c}{ Cooling } & \multicolumn{2}{c}{ Control } & \multicolumn{2}{c}{ Cooling } \\
& $\mathbf{R}$ & $\boldsymbol{p}$ & $\mathbf{R}$ & $\boldsymbol{p}$ & $\mathbf{R}$ & $\boldsymbol{p}$ & $\mathbf{R}$ & $\boldsymbol{p}$ & $\mathbf{R}$ & $\boldsymbol{p}$ & $\mathbf{R}$ & $\boldsymbol{p}$ \\
\hline Thirst & 0.079 & 0.827 & -0.045 & 0.902 & $\sim$ & $\sim$ & $\sim$ & $\sim$ & $\sim$ & $\sim$ & $\sim$ & $\sim$ \\
RPE & 0.610 & 0.061 & 0.531 & 0.115 & 0.256 & 0.476 & -0.297 & 0.405 & $\sim$ & $\sim$ & $\sim$ & $\sim$ \\
Tgi & -0.059 & 0.872 & -0.210 & 0.561 & 0.086 & 0.812 & -0.451 & 0.191 & -0.026 & 0.944 & 0.348 & 0.325 \\
Tsk & -0.060 & 0.870 & -0.083 & 0.819 & 0.108 & 0.766 & -0.030 & 0.933 & 0.465 & 0.176 & 0.013 & 0.972 \\
HR & -0.502 & 0.139 & 0.050 & 0.891 & 0.046 & 0.900 & 0.350 & 0.322 & -0.052 & 0.886 & 0.161 & 0.657 \\
\hline
\end{tabular}

No significant relationships were found.

Note. $\mathrm{RPE}=$ rating of perceived exertion, $\mathrm{T}_{\mathrm{gi}}=$ core body temperature, $\mathrm{T}_{\mathrm{sk}}=$ skin temperature, and $\mathrm{HR}=$ heart rate 


\begin{tabular}{|c|c|c|c|c|c|c|c|}
\hline $10 \mathrm{~min}$ & $20 \mathrm{~min}$ & $\begin{array}{c}5 \text { min } \\
\text { Break } \\
1\end{array}$ & $20 \mathrm{~min}$ & $\begin{array}{c}5 \text { min } \\
\text { Break } \\
2\end{array}$ & $20 \min$ & $\begin{array}{c}5 \text { min } \\
\text { Break } \\
3\end{array}$ & $30 \mathrm{~min}$ \\
\hline $\begin{array}{l}\text { Warm- } \\
\text { Up } \\
\text { Data } \\
\text { collected } \\
\text { Pre- and } \\
\text { Post }\end{array}$ & $\begin{array}{l}\text { Anaerobic } \\
\text { Exercise }\end{array}$ & 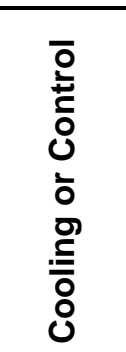 & $\begin{array}{c}\text { Anaerobic } \\
\text { Exercise }\end{array}$ & 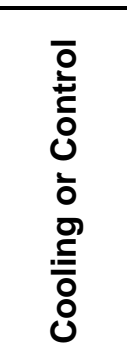 & $\begin{array}{c}\text { Anaerobic } \\
\text { Exercise }\end{array}$ & 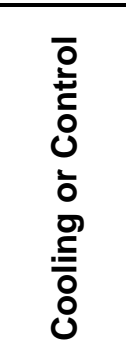 & $\begin{array}{l}\text { Recovery } \\
\text { Data collected } \\
\text { every } 5 \text { min }\end{array}$ \\
\hline$\widehat{T}$ & & & & & & & \\
\hline
\end{tabular}

Figure 1. Research design, 代indicates data collection time points. 


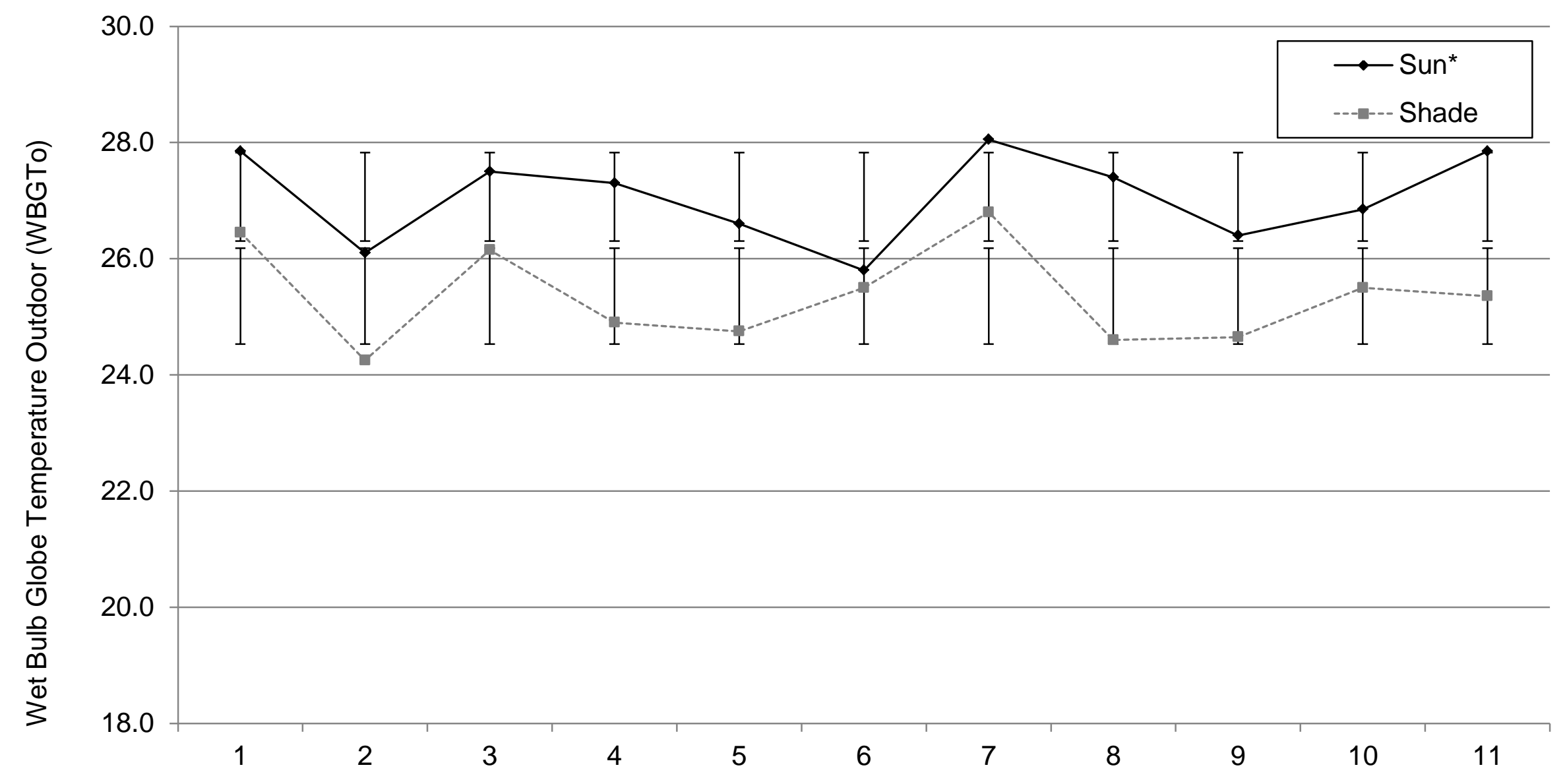

Days of Data Collection

Figure 2. Environmental conditions in the sun and in the shade (11 days)

$\mathrm{WBGT}_{0}=$ wet bulb globe temperature outdoors

${ }^{*}$ Significantly higher than Shade $(p<0.05)$ 


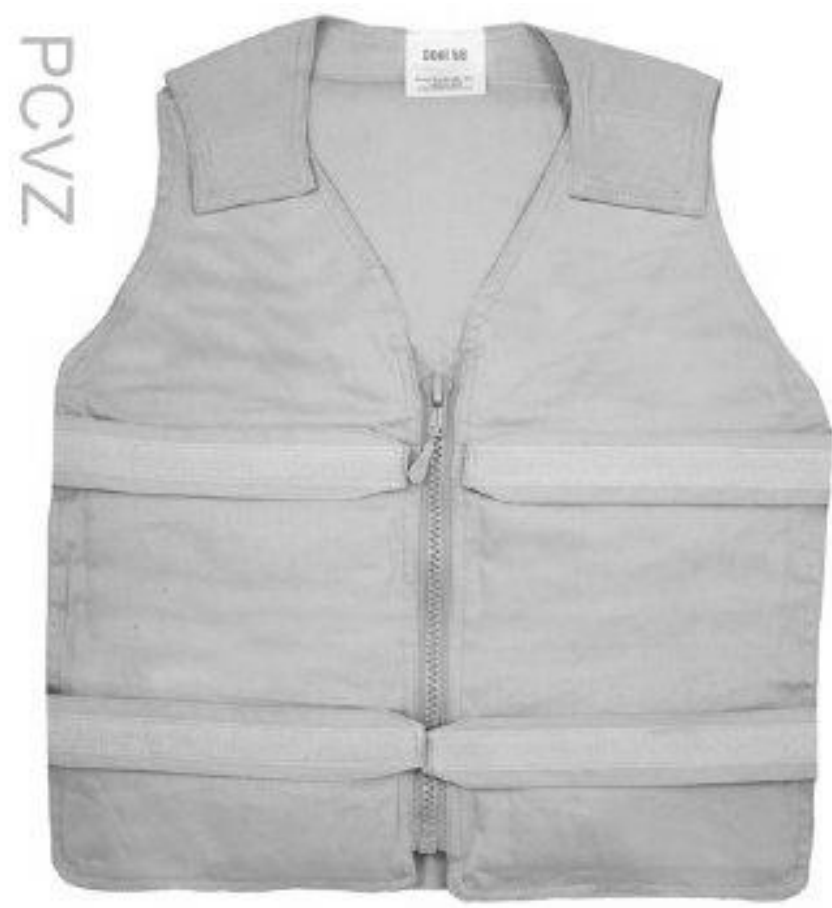

Figure 3. Polar Products Cool58 ${ }^{\mathrm{TM}}$ Phase Change Vest.

Retrieved from:

http://www.polarproducts.com/polarshop/pc/Cool58-Phase-Change-Zipper-Vest16p197.htm. Accessed on April 20, 2013. 


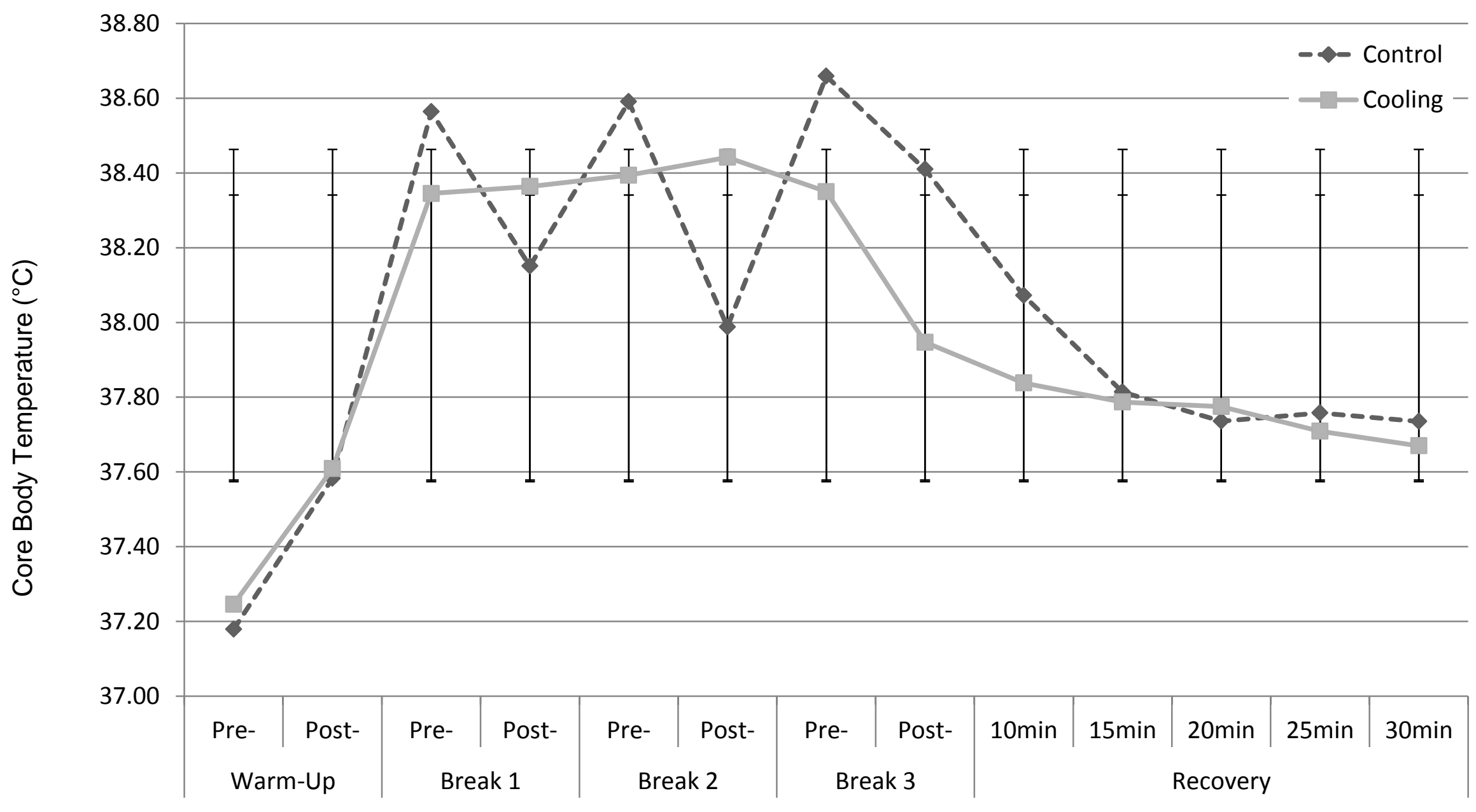

\section{Exercise Breaks and Recovery}

Figure 4. Core Temperature for Intermittent Cooling during Exercise and Recovery $(n=10$, mean $\pm S D)$

For $\mathrm{T}_{\mathrm{gi}}$ during exercise we found no significant differences between conditions ( $\left.p=.674\right)$; however we did find a significant decrease in $\mathrm{T}_{\mathrm{gi}}$ during Break 3 (pre-test $=38.50 \pm .13^{\circ} \mathrm{C}$ vs. post-test $=38.18 \pm .13^{\circ} \mathrm{C}$ ) for both conditions. For $\mathrm{T}_{\mathrm{gi}}$ during recovery, we found significant differences between condition ( $p=.452$ ) and $\mathrm{T}_{\mathrm{gi}}$ progressively decreased during recovery $(p<.001)$. For the $\Delta \mathrm{T}_{\mathrm{gi}}$ we found no significant differences for condition $(p=.152)$. 


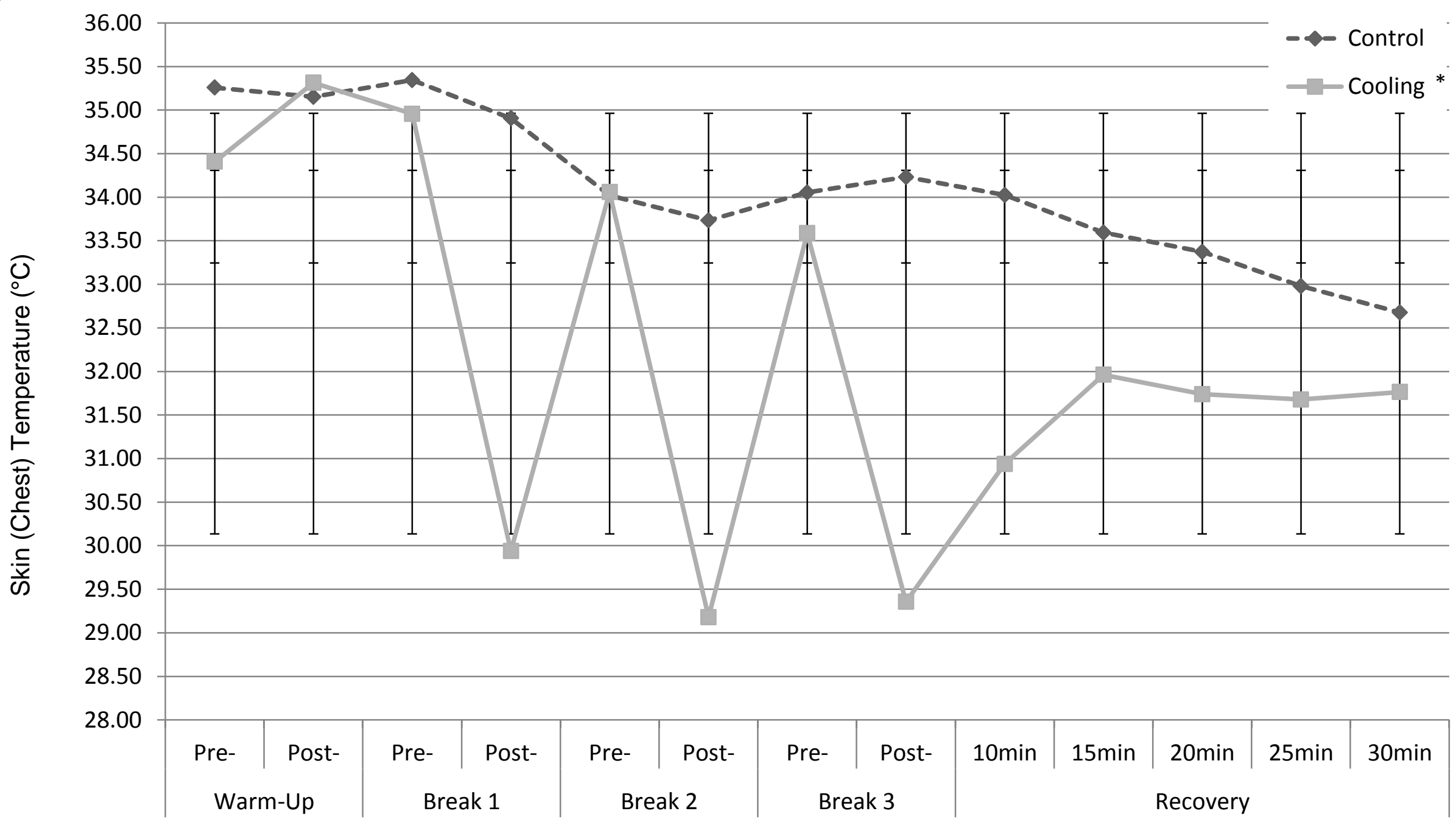

\section{Exercise Breaks and Recovery}

Figure 5. Skin (chest) Temperature for Intermittent Cooling during Exercise and Recovery $(n=10$, mean $\pm S D)$

*During exercise we found significantly $(p<0.001)$ decreased skin temperature for the Cooling condition $\left(\mathrm{T}_{\text {chest }}=31.85 \pm 0.43^{\circ} \mathrm{C}\right)$ compared to the Control condition $\left(\mathrm{T}_{\text {chest }}=34.38 \pm 0.43^{\circ} \mathrm{C}\right.$ ). During recovery, we found significantly $(p<0.001)$ lower skin temperature in the Cooling condition $\left(T_{\text {chest }}=31.24 \pm 0.47^{\circ} \mathrm{C}\right)$ compared to the Control condition $\left(T_{\text {chest }}=33.48 \pm 0.47^{\circ} \mathrm{C}\right)$. 


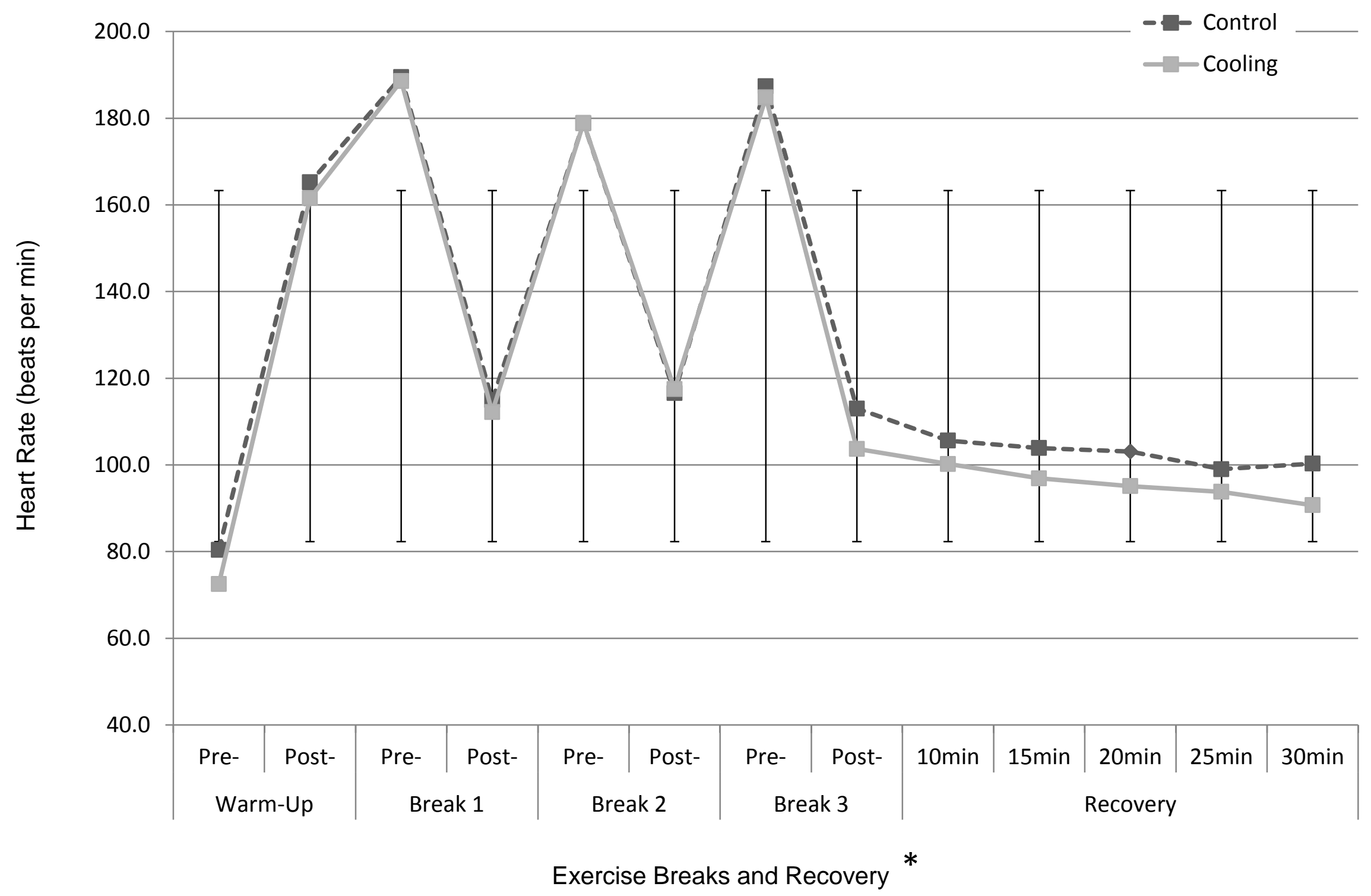

Figure 6. Heart Rate for Intermittent Cooling during Exercise and Recovery ( $n=10$, mean $\pm S D$ )

During exercise we found no significant difference ( $p=0.586$ ) between the Cooling condition (HR=147.6 $\pm 2.6 \mathrm{bpm}$ ) and the Control condition $(H R=150.1 \pm 3.5 \mathrm{bpm})$; however we did find *significant differences between pre- and post-test $(p<0.001)$. During recovery, we found no significant $(p=0.229)$ differences between conditions; however we did find *significant differences between pre- and post-test $(p<0.001)$. 


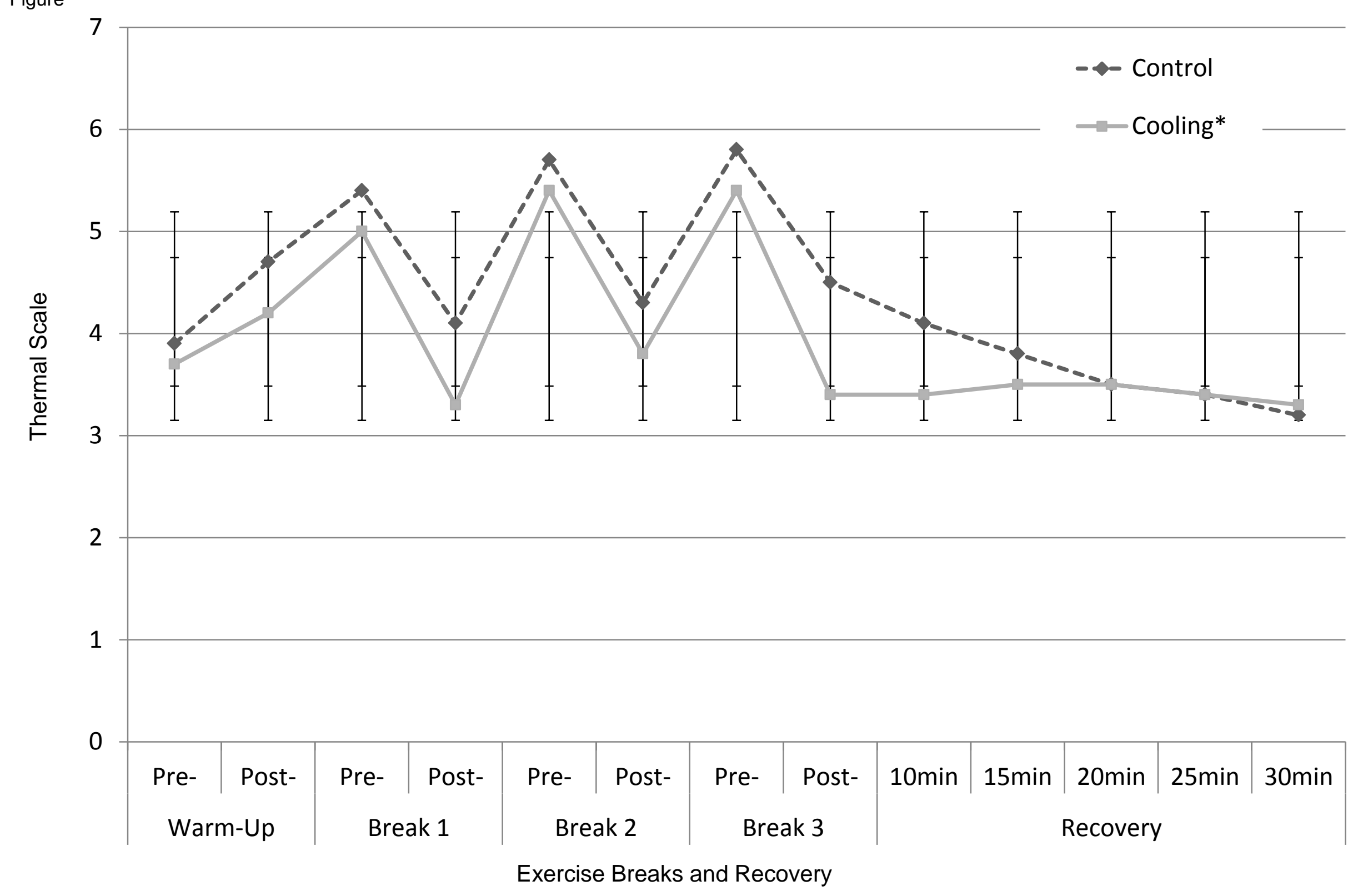

Figure 7. Thermal responses for Intermittent Cooling during Exercise and Recovery $(n=10$, mean $\pm S D)$

${ }^{*}$ Significantly $\left(F_{1,18}=43.038, p=0.026\right)$ lower for the Cooling condition ( $4.4 \pm 0.2$ points) compared to the Control condition (5.0 \pm 0.2 points). 


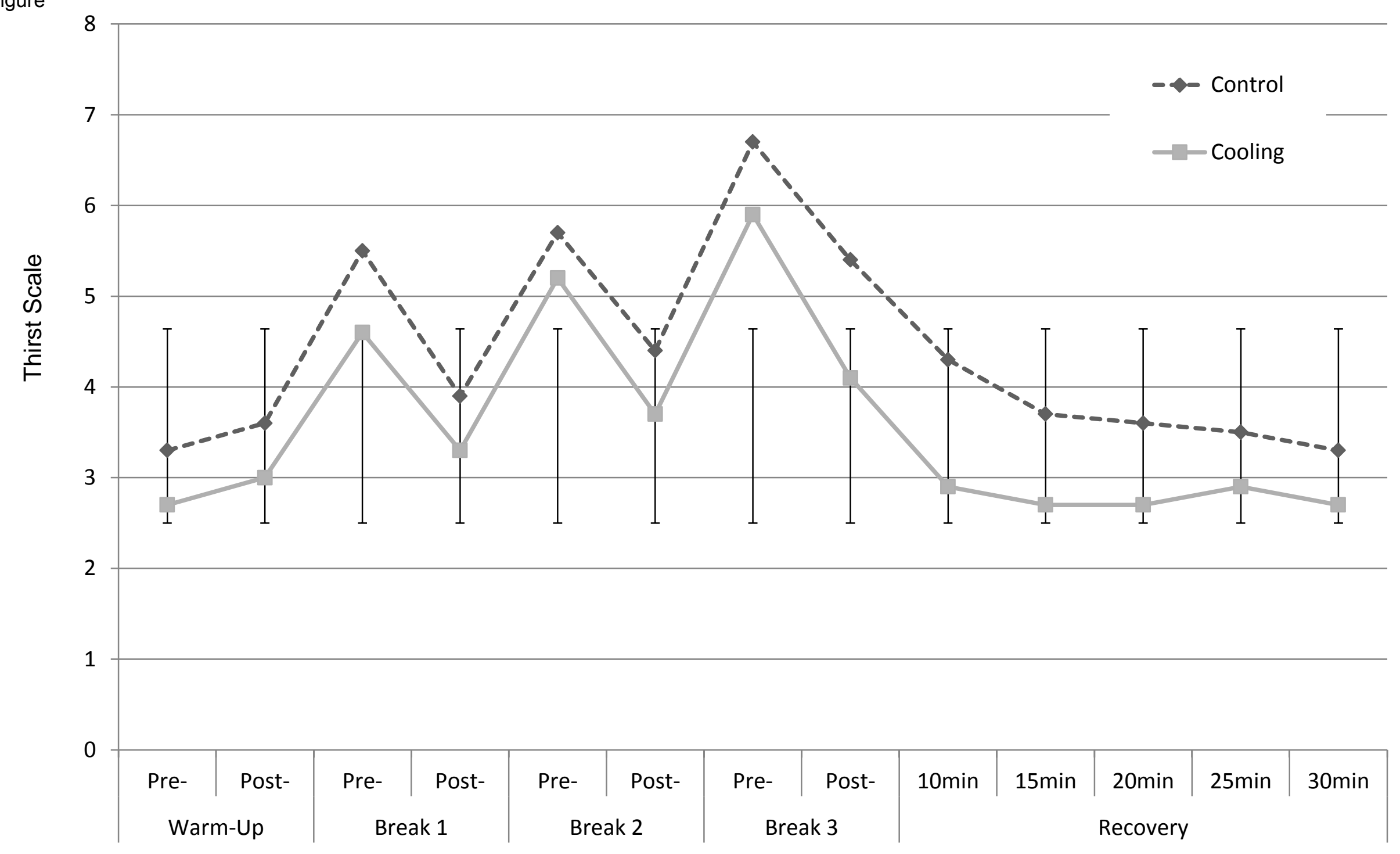

\section{Exercise Breaks and Recovery}

Figure 8. Thirst responses for Intermittent Cooling during Exercise and Recovery $(n=10$, mean $\pm S D)$ Approached significance $(p=0.051)$ with the Cooling condition lower $(4.467 \pm 0.288$ points) than the Control condition $(5.267 \pm 0.392$ points). 
Man乡̧scripゆ ( NO AUTHOR INFORMATION-Manuscript Text Pages, including References and Figure Legends)

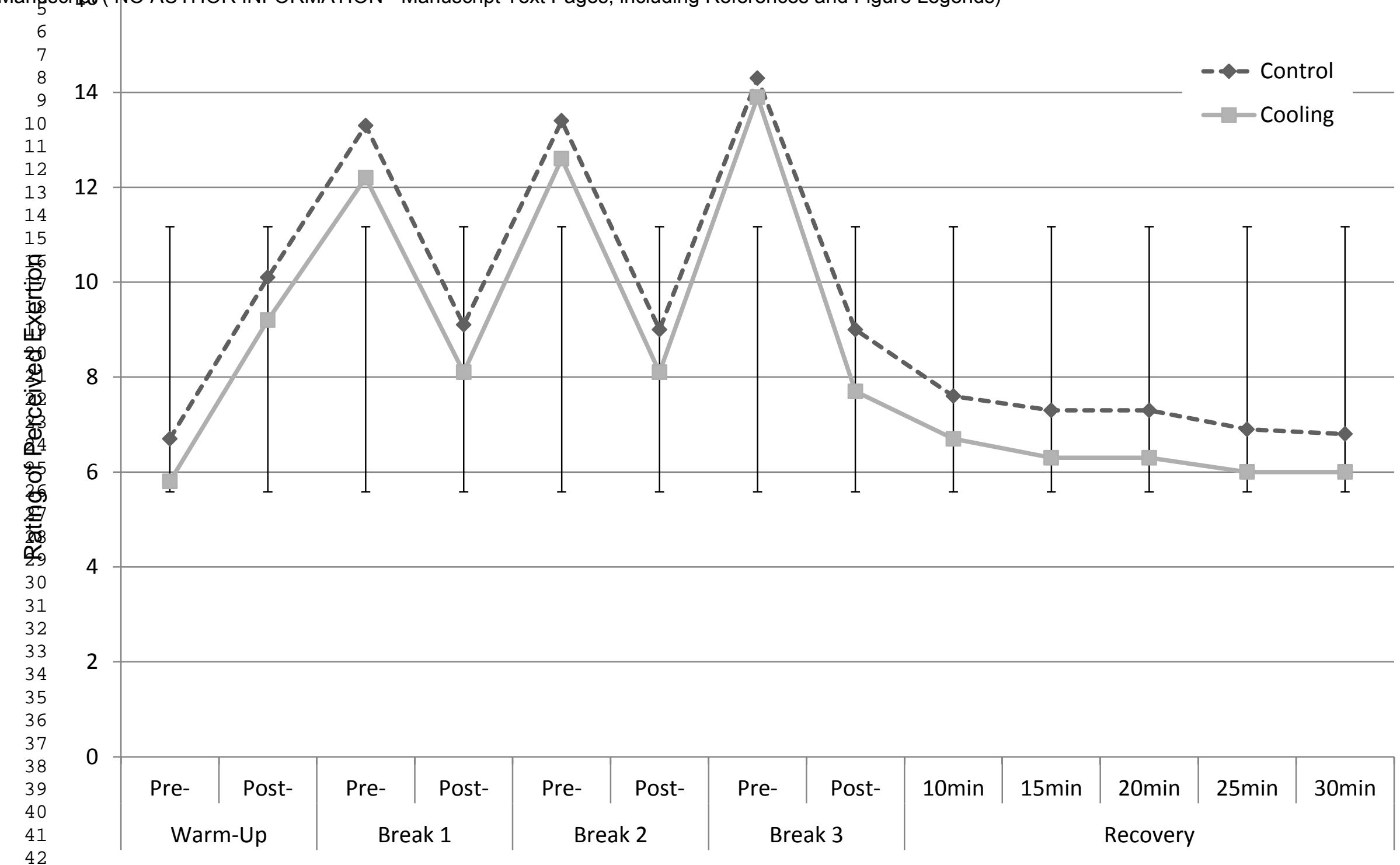

\section{Exercise Breaks and Recovery}

46

47 Figure 9. RPE responses for Intermittent Cooling during Exercise and Recovery ( $n=10$, mean $\pm S D)$

48 No significant $(p=0.164)$ differences between conditions. 